INPLASY PROTOCOL

To cite: Carvalho et al. Instruments for assessing loneliness in older people in Portugal: a Scoping Review protocol. Inplasy protocol 2021100002. doi:

10.37766/inplasy2021.10.0002

Received: 01 October 2021

Published: 01 October 2021

Corresponding author:

Rita Carvalho

rita.carvalho@ua.pt

Author Affiliation:

University of Aveiro

Support: No financial support.

Review Stage at time of this submission: Preliminary searches.

Conflicts of interest: None declared.

\section{Instruments for assessing loneliness in older people in Portugal: a Scoping Review protocol}

\author{
Carvalho, R'; Tavares, J2; Sousa, L³.
}

Review question / Objective: The objective of this scoping review is to map the instruments validate for the Portuguese older population (65+ years old) that assess loneliness; and to identify their psychometric properties and contexts where they have been in use. The questions for this scoping review are: What are the validated instruments for Portugal that assess loneliness in the older individuals? What are the psychometric properties of those instruments? In which contexts were the loneliness assessment instruments used?

Eligibility criteria: Participants - This scoping review will consider all studies that included older adults with 65 years and over. Concept - This review will be included studies that assess loneliness or cover loneliness by validated instruments that address different dimensions, including, but not limited to, emotional or social. Context - This scoping review will consider studies that used validated instruments the loneliness in Portuguese older adults including, but not limited to the context of community, intermediate care, long-term care or acute care. Types of sources - This scoping review will consider quantitative and mixed-method studies. In the quantitative designs include experimental, quasi-experimental, observational and analytical observational studies including prospective and retrospective cohort studies, case-control studies, and analytical crosssectional studies will be considered for inclusion. This review will also consider descriptive observational study designs including case series, individual case reports, and descriptive cross-sectional studies for inclusion.

INPLASY registration number: This protocol was registered with the International Platform of Registered Systematic Review and Meta-Analysis Protocols (INPLASY) on 01 October 2021 and was last updated on 01 October 2021 (registration number INPLASY2021100002).

\section{INTRODUCTION}

Review question / Objective: The objective of this scoping review is to map the instruments validate for the Portuguese older population $(65+$ years old) that assess Ioneliness; and to identify their psychometric properties and contexts 
where they have been in use. The questions for this scoping review are: What are the validated instruments for Portugal that assess loneliness in the older individuals? What are the psychometric properties of those instruments? In which contexts were the loneliness assessment instruments used?

Background: Demographic aging is a worldwide phenomenon portrayed by the increase in life expectancy and the decline in birth and fertility rates (Moreira, 2020). Data from Eurostat (2020) show Portugal as the fourth most aged country in the European Union. Despite the rates of mortality due to Covid-19 virus, the Portuguese National Institute of Statistics (INE, 2021), estimates that the demographic aging will continue to increase. With the increase in the older population and due to the Covid-19 pandemic, interest in the study of loneliness has also increased, as loneliness has been described as increasing in this age group. At the beginning of the pandemic, between April and July 2020, loneliness in the European Union doubled; and Portugal was the sixth country with the highest increase; compared to prepandemic time, loneliness in Portugal increased by more than 15 percentage (European Commission, 2021). Some factors related to the transition to old adulthood tend to be associated to increased risk of loneliness. In particular, retirement (that leads loss of social status and some social relations) and loss of friends and relatives (the older ones die or lose functionality; some of the youngers migrate) are features of emotional distress. In these circumstances, older adults face the potential shrink of their social networks, both in structural and functional terms, putting them at increased risk for Ioneliness and/or social isolation (Nicolaisen \& Thorsen, 2017). Loneliness is a strong subjective and negative experience. It is the perception of a discrepancy between a person's desired and actual network of relationships. It is not only about having too few social contacts, but also about the perception that these relationships are not satisfying.
In other words, loneliness does not mean being alone, but feeling alone (European Comission, 2021). Weiss (1973) considerers two types of loneliness: emotional and social. The emotional loneliness results from the lack of a close, intimate attachment to another person, and is often experienced by people who have recently been divorced, widowed, or ended a dating relationship. The social loneliness is associated to the lack of a social network in which the person is part of a group sharing common interests and activities. This last type is commonly experienced by individuals who have recently moved to a new social environment (such as a new city, job, or college) (Russel, Cutrona, Rose \& Yurko, 1984). The Covid-19 pandemic was all about isolation and distance, thus increasing loneliness, especially in older adults, since they were the ones who demanded more protection due to the changes in the human immune system as we age, that makes it more difficult for the body to respond to viral infection. Therefore, it is essential to use instruments that can identify cases of loneliness and describe the loneliness in populations. International literature presents a set of instruments, most Likert scales, that assess loneliness. Most scales are unidimensional (measures how lonely a person is), while others are more multidimensional (measures how lonely a person is and what kind of loneliness they are experiencing) (Harvey \& Walsh, 2016). Preventing loneliness is a public health priority. Portugal is an aged country, highly affected by Covid-19 particularly in older individuals. Therefore, it is crucial to have measures of loneliness validated for our population.

Rationale: People are living longer, and the population aged 65 and over is increasing, with Europe presenting one of the highest proportions of older adults (United Nations Department of Economic and Social Affairs, 2019). Literature shows no direct relations between loneliness and old age, but there are personal and social factors that make older adults more vulnerable to Ioneliness (Freitas, 2011). Some individual factors include living alone, limited family 
relations or support, disruptive life events (such as a move to a different residence), bereavement, illness and poor health and functional disability (which limits a person's ability to socialize), sensory deprivation (such as hearing loss), and retirement (Donovan \& Blazer, 2020). Different definitions of loneliness have been proposed by various investigators to understand its nature. However, all definitions seem to involve three assumptions: i) loneliness is subjective; ii) loneliness is an unpleasant and distressing experience; iii) loneliness is manifested when there is a discrepancy between a person's actual and desired social needs (Peplau \& Perlman, 1982). Although loneliness and social isolation are two distinct concepts, social isolation reflects a condition that may contain elements of loneliness or aloneness, depending on whether choice is involved. In simple terms: social isolation by choice is aloneness, and without choice is loneliness. Differentiating Another concept worsening clarification is alienation; alienation refers to the objective or physical separation from someone or something that one is attached to; Ioneliness refers to the subjective experience of separation from that person or thing. Solitude is another borderline concept; it isa refreshing and calming state, that can be seen when people "take time out", indicating freedom of choice, and useful in coping with loneliness (Bekhet \& Zauszniewski, 2008). When the feeling of loneliness extends in time, it can compromise well-being, quality of life, physical health, and, often leading to less time of life (Hawkley \& Cacioppo, 2010; Luanaigh \& Lawlor, 2008). Assessing loneliness with validate instruments is crucial to the early diagnosis and individual intervention, as well as for community characterization and intervention. Assessment is relevant so that adequate strategies can be developed to minimize the detrimental consequences of loneliness (Kznier, Oliveira, Mata \& Chianca, 2016). To adequately assess loneliness, it is essential to use reliable instrument, validate for the specific population and context. A preliminary search of JBI Database of
Systematic Reviews and Implementation Reports, the Cochrane Library and MEDLINE was conducted, and no scoping review (published or in progress) related to instruments validated for Portuguese population that assess loneliness were identified. This scoping review will identify the instruments validated for the older Portuguese population and will provide guidance for researchers and practitioners for the selection of the best instrument to measure loneliness. This scoping review aims to identify and map the instruments validated for Portugal that assess loneliness in people aged 65 and over.

\section{METHODS}

Search strategy: The proposed scoping review will be conducted in accordance with JBI methodology for scoping reviews. The search strategy aims to locate published and unpublished studies. An initial search of MEDLINE (PubMed) and Scopus was undertaken to identify articles on the topic. The title, abstract, and keywords was analysis to find the text words that will be used to build a full search strategy. The databases to be searched will include SciELO, Psyclnfo, Scopus, MEDLINE (PubMed), MedicLatina (EBSCO), Nursing \& Allied Health Collection: Comprehensive (EBSCO) and CINAHL (EBSCO). Source of unpublished studies/ grey literature to be searched will include RCAAP (Open Access Scientific Repositories of Portugal). The reference lists of articles included in the review will be screened. The studies identify will be assess for relevance based on the title and abstract of the papers. Studies published in Portuguese, Spanish, and English from 1978 onwards will be considered for inclusion. The application of this time limitation is because the UCLA loneliness scale was originally launched that year (with a 20-item scale) (Russell, Peplau \& Ferguson, 1978).

Eligibility criteria: Participants - This scoping review will consider all studies that included older adults with 65 years and over. Concept - This review will be included studies that assess loneliness or cover 
loneliness by validated instruments that address different dimensions, including, but not limited to, emotional or social. Context - This scoping review will consider studies that used validated instruments the loneliness in Portuguese older adults including, but not limited to the context of community, intermediate care, long-term care or acute care. Types of sources - This scoping review will consider quantitative and mixed-method studies. In the quantitative designs include experimental, quasi-experimental, observational and analytical observational studies including prospective and retrospective cohort studies, case-control studies, and analytical cross-sectional studies will be considered for inclusion. This review will also consider descriptive observational study designs including case series, individual case reports, and descriptive cross-sectional studies for inclusion.

Source of evidence screening and selection: Following the search, all identified citation will be collated and uploaded into Mendeley V1.19.8 and duplicates removed. The articles will be imported into the Rayyan software. Then, titles and abstracts will be reviewed by two independent reviewers (RC and LS) for assessment against the inclusion criteria for the scoping review. In case of doubts about the relevance of the study or if the abstract is not explicit, the full article will be retrieved. Studies that could potentially meet the inclusion criteria will be retrieved in full text and assess in detail against the criteria inclusion. Full text studies that do not meet the inclusion criteria will be excluded, and reasons for exclusion will be provided in the scoping review. Any disagreements will be resolved through discussion, and if consensus is not reached, disagreements will be resolved with a third reviewer. The results of the search will be report in full in the final scoping review and presented in a Preferred Reporting Items for Systematic Reviews and Meta-Analyses Extension for Scoping Reviews (PRISMA-ScR) flow diagram.
Data management: Data will be extracted from papers included in the scoping review by two independent reviewers using the draft data extraction tool. The data extracted will include specific details like the authors, year of publication, country of origin, aims, type of study (instrument development and initial validation, validation of adapted or translated instrument, validation in a new population) and instrument title and/or abbreviation. To assess the Psychometric properties of instruments, a data extraction worksheet will be developed to: 1) describe the process or translation, adaptation and validation theoretical framework, 2) the concept/construct the instrument operationalizes, 3) sample and context where the instrument was used, 4) administration method, 5) description of items, 6) quotation and interpretation, 7) reliability, and 8) validity. The draft data extraction tool will be modified and revised as necessary during the process of extracting data from each included study. These modifications will be detailed in the full scoping review report. Any disagreements that arise between the reviewers will be resolved through discussion or with a third reviewer.

Reporting results / Analysis of the evidence: The results of the search will be reported in full in the final manuscript and presented in a Preferred Reporting Items for Systematic Reviews and Meta-Analyses Extension for Scoping Reviews (PRISMAScR) flow diagram.

Presentation of the results: The extracted data will be presented in tabular form in a manner that aligns with the review questions. A narrative summary will accompany the tabulated results and will describe how the results relate to the review's objective and questions.

Language: English, Portuguese, and Spanish.

Country(ies) involved: Portugal.

Keywords: Aging; older; loneliness; instruments; scales, psychometric 
propriety, reliability; validation; Portugal; Portuguese.

Contributions of each author:

Author 1 - Rita Carvalho.

Email: rita.carvalho@ua.pt

Author 2 - João Tavares.

Email: joaoptavares@ua.pt

Author 3 - Liliana Sousa.

Email: lilianax@ua.pt 